\title{
Juvenile Dermatomyositis—Clinical Phenotypes
}

\author{
Danyang Li, PhD ${ }^{1}$. Sarah L Tansley, PhD, MRCP ${ }^{1}$
}

Published online: 11 December 2019

(C) The Author(s) 2019

\begin{abstract}
Purpose of Review Juvenile dermatomyositis is a heterogeneous disease with variable clinical outcomes. Here, we describe the recognised subtypes of idiopathic inflammatory myositis which occur in children, with particular reference to disease-associated autoantibodies.

Recent Findings Large cohort studies have demonstrated that myositis autoantibodies are common in juvenile dermatomyositis and can be found in the majority of patients. They identify homogenous clinical subgroups and inform prognosis, particularly the risks of developing interstitial lung disease. Descriptions of immune-mediated necrotising myositis in juvenile patients have highlighted a rare but important clinical subset typically associated with severe muscle disease and treatment resistance.

Summary It is increasingly apparent that autoantibodies can provide detailed information on prognosis and the likely disease associations in those with juvenile dermatomyositis. Further work is needed to establish how this knowledge should influence our approach to treatment.
\end{abstract}

Keywords Juvenile $\cdot$ Myositis $\cdot$ Autoantibodies $\cdot$ Subgroup $\cdot$ Phenotype

\section{Introduction}

Juvenile-onset myositis is a very rare disease with an incidence of approximately $2-4$ per million $[1,2]$. The vast majority of affected children have associated cutaneous disease, and, as such, juvenile dermatomyositis (JDM) is often used as an umbrella term for all juvenile-onset myositis. Within the JDM subgroup, there remains significant heterogeneity with variable chronicity, organ involvement and long-term clinical outcome. The Bohan and Peter criteria, proposed in 1975 [3, 4], have been widely used to identify patients; however, they fail to describe the full spectrum of myositis and provide with no definition for clinically amyopathic myositis, immunemediated necrotising myositis or inclusion body myositis; although the latter is not seen in children. In 2017, the European League Against Rheumatism and American College of Rheumatology published new classification criteria for adult

This article is part of the Topical Collection on Inflammatory Muscle Disease

Sarah L Tansley, PhD, MRCP

s.tansley@nhs.net

1 University of Bath, Claverton Down, Bath BA2 7AY, UK and juvenile idiopathic inflammatory myopathies and their major subgroups. Limitations of the updated criteria include the exclusion of myositis-specific autoantibodies, except antiJo-1, and histological features relevant to immune-mediated necrotising myositis (IMNM) [5].

Strategies to better define homogeneous subgroups of patients with JDM are crucial to facilitate diagnosis, inform prognosis and permit good-quality clinical trials of novel and existing therapies. Recent studies have demonstrated that autoantibodies can be identified in $60-95 \%$ of patients with JDM and provide a further degree of phenotypic refinement $[6 \bullet \bullet, 7,8]$, in addition to histopathological correlations [9]. Here, we discuss the different clinical phenotypes of patients with JDM and suggest autoantibody status as a means of identifying key subgroups, which is summarised in Table 1.

\section{Classic Dermatomyositis}

Anti-Mi2 is generally considered the archetypal dermatomyositis autoantibody. Affected patients typically present with significant skin and muscle involvement and have a low incidence of additional organ involvement. These patients have histologically severe disease on muscle biopsy with high 

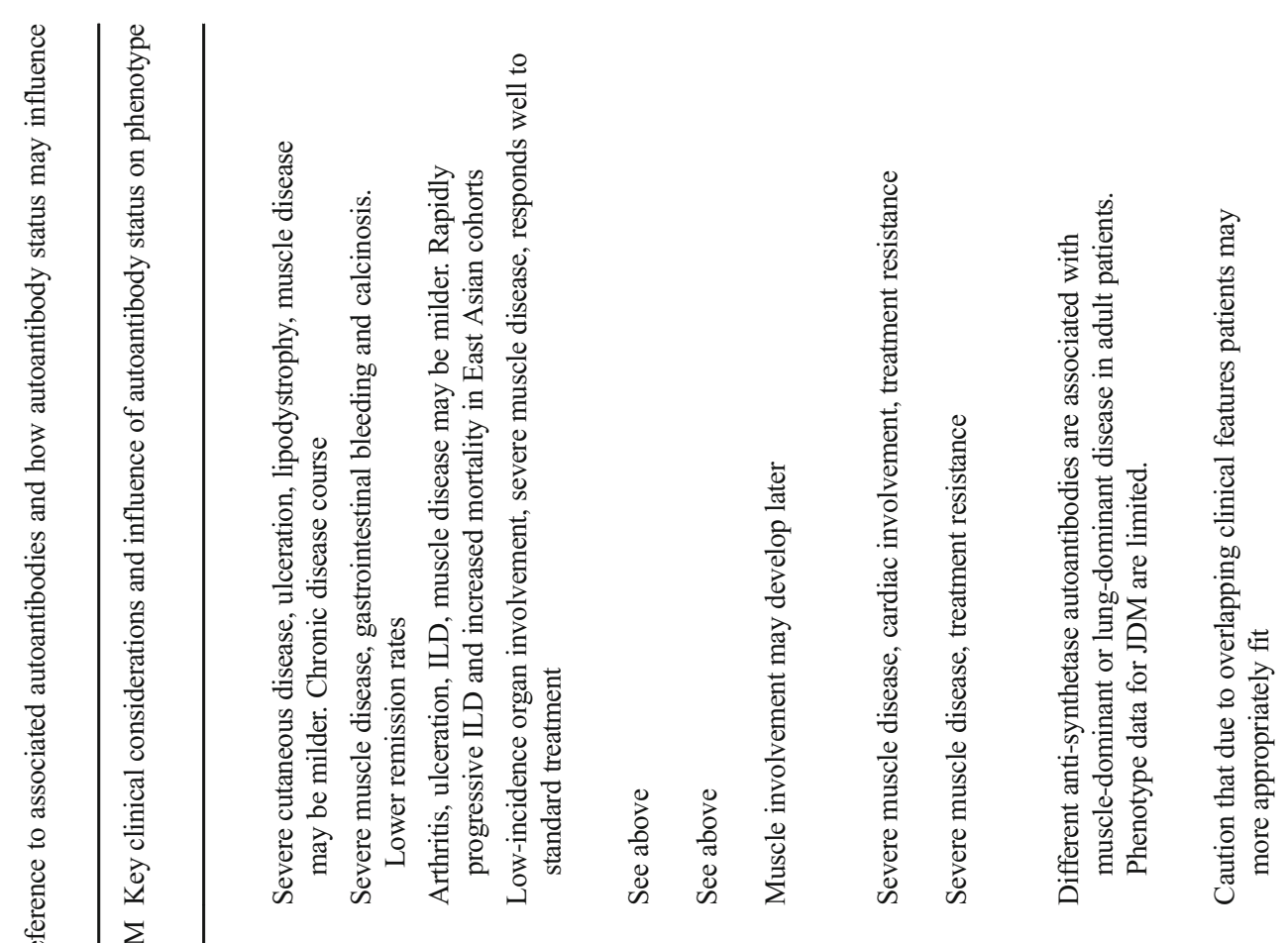

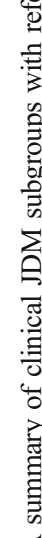

in

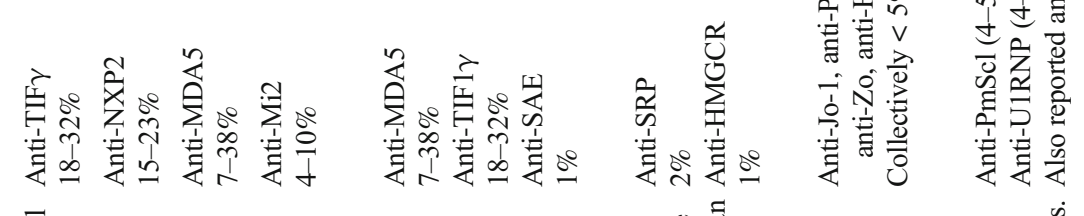

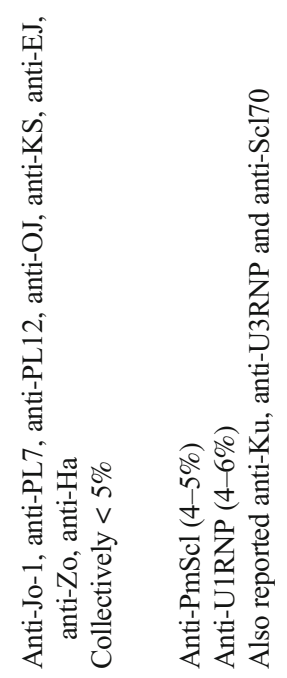

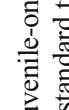

密跑

言学

递兽

氙,
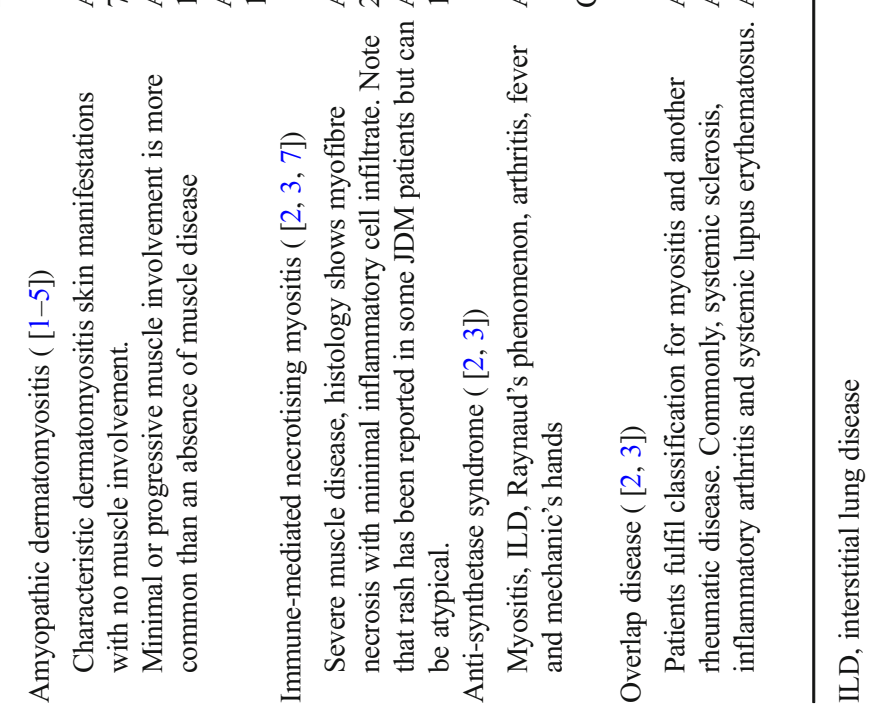
overall severity scores and characteristic histological features [10•]. Interestingly, while anti-Mi2-associated JDM is often severe at presentation, these patients typically respond well to conventional therapy and are considered to have good prognosis $[6 \bullet \bullet]$.

Most patients with JDM who present with classic dermatomyositis will not have anti-Mi2 disease as this autoantibody is found in just $4-10 \%$ of affected patients [6••]. While often presenting similarly with both skin and muscle involvement, patients with anti-NXP2 (15-23\% of patients) and anti-TIF $\gamma$ (18-32\% of patients) are more likely to be resistant to conventional therapies $[6 \bullet \bullet, 7,11]$.

Patients with anti-NXP2 have more severe muscle disease $[6 \bullet \bullet, 8,11,12]$ in addition to an increased risk of gastrointestinal bleeding, ulcers and dysphagia [7]. Disease outcome also appears to be worse in this group with more persistent disease activity and a worse functional status [7, 11, 12]. A recent study exploring the clinico-pathological subgroups of JDM found that anti-NXP2 occurred more often in a subgroup of patients with severe muscle weakness, gastrointestinal involvement and prominent severe ischaemic features on muscle biopsy [13]. Furthermore, patients with anti-NXP2 required treatment that was more aggressive and had a lower remission rate during the follow-up period [13]. Calcinosis is a recognised complication of dermatomyositis that is more frequently seen in juvenile-onset disease. Anti-NXP2 autoantibodies have been associated with the development of calcinosis in UK patients, but the association bordered on significance when a larger group of patients was analysed, and this has not been replicated in US studies [6••, 7, 14]. Calcinosis is a cause of significant morbidity, and, in addition to autoantibody status, a recent study using the CARRA legacy registry has linked calcinosis to prolonged active disease, severe disease and clinical features, such as lipodystrophy and joint contractures [15]. Whether anti-NXP2 autoantibodies are directly associated with calcinosis or prolonged active disease leading to calcinosis remains to be seen, but there are increasingly strong arguments for managing such patients aggressively.

In contrast, patients with anti-TIF1 $\gamma$ may have milder muscle involvement $[6 \bullet \bullet, 7]$. This subgroup has been associated with more severe cutaneous disease, including ulceration, and, in US studies, lipodystrophy, a late complication of JDM and associated with a severe, chronic disease course [7, 8, 16, 17]. In the UK, patients with anti-TIF1 $\gamma$ are more likely to receive treatment with IV cyclophosphamide, typically reserved for patients with severe disease, although the clinical drivers behind treatment choices remain unknown [6••]. Adult myositis patients with anti-TIF1 $\gamma$ are at increased risk of malignancy within three years of diganosis, but this association is not seen in JDM [6••, 18].

Anti-MDA5 autoantibodies have also been described in JDM, and affected patients typically have milder muscle disease, both clinically and histologically [19]. Importantly, this group has an increased likelihood of developing interstitial lung disease in addition to ulceration and arthritis [19-22]. The prevalence of anti-MDA5 is variable and appears to depend on ethnicity and/or place of origin with $7 \%$ of UK children having this autoantibody compared to up to $38 \%$ in Japanese JDM cohorts [8, 19, 21, 22]. Rapidly progressive interstitial lung disease and a high associated mortality have been reported in juvenile East-Asian cohorts with anti-MDA5 but not in a predominantly Caucasian UK cohort [19, 21, 22]. A recent US study suggested that the co-existance of antiMDA5 with anti-Ro52 autoantibodies in JDM patients significantly increased the risk of interstitial lung disease which developed in $9 \%$ of those with anti-MDA5 and $70 \%$ of those with anti-MDA5 and anti-Ro52 [23••]. One patient with both autoantibodies developed rapidly progressive interstitial lung disease $[23 \bullet \bullet$. The identification of anti-MDA5 autoantibodies, particularly in conjunction with anti-Ro52, should prompt careful monitoring for the development of interstital lung disease.

Thus, while anti-Mi2 patients are likely to be best managed by standard conventional therapies, those with anti-NXP2 and anti-TIF1 $\gamma$ may benefit from an alternative, more aggressive treatment approach. Patients with anti-MDA5 autoantibodies should be carefully monitored for the development of interstitial lung disease.

\section{Amyopathic Myositis}

Amyopathic or clinically amyopathic dermatomyositis describes those patients with characteristic dermatomyositis skin manifestations in the absence of muscle involvement, as defined by clinical examination, muscle enzymes and muscle biopsy. Amyopathic myositis is recognised in children, but it is rare, and, more commonly, patients have mild or progressive muscle involvement $[24,25]$. The relationship between skin and muscle disease is complex; patients may present with skin disease alone and subsequently develop muscle involvement, a pattern which has been described in adults and chil-

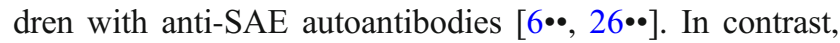
those who initially have muscle dominant disease may later have persistent skin disease despite remission of muscle disease. Residual skin disease in JDM is now recognised as important and is associated with a longer time to remission [27]. Skin changes should be regularly monitored as part of standard care and actively treated, even in the absence of muscle disease activity [27].

Anti-MDA5 autoantibodies are associated with amyopathic myositis in adult and juvenile cohorts [19-22]. Anti-TIF $1 \gamma$ has also been reported in patients with amyopathic and hypomyopathic myositis but to a lesser degree and mainly in adult patients [28, 29]. In a small Japanese 
cohort of patients with JDM, muscle weakness was absent in $40 \%$ of anti-MDA 5 patients and $44 \%$ of anti-TIF1- $\gamma$ at presentation; however, all but one patient with anti-MDA5 autoantibodies developed muscle involvement during follow-up [8].

Dermatomyositis skin changes in the absence of or associated with minimal muscle disease should prompt investigation for myositis autoantibodies. The identification of anti-MDA5 autoantibodies can be helpful in identifying those patients at increased risk of interstitial lung disease, and further investigation should be considered. Patients with amyopathic JDM frequently develop muscle involvement at a later stage.

\section{Immune-Mediated Necrotising Myositis}

IMNM is a relatively recently described subtype of myositis characterised by myofibre necrosis, with minimal inflammatory cell infiltrate on muscle biopsy [30]. In those with adult-onset myositis, most patients previously labelled as polymyositis can now be classified as either IMNM or anti-synthetase syndrome. Polymyositis is rare in those with juvenile-onset disease and occurs in less than $8 \%$ of patients [31]. Unlike adults, skin disease has been reported in JDM patients who otherwise present as IMNM and should not prevent this diagnosis from being considered. When present associated rashes are often atypical [6••], IMNM typically presents with severe muscle weakness and markedly elevated muscle enzymes. It is associated with autoantibodies directed against signal recognition peptide (SRP) and HMG-CoA reductase (3-hydroxy-3-methyl-glutaryl-coenzyme A reductase (HMGCR), and patients are often resistant to standard therapies.

Anti-SRP autoantibodies are well-described in adult patients, but studies involving children are generally small. Anti-SRP can be found in approximately $2 \%$ of JDM patients who can be expected to present with severe weakness, minimal or no skin rash and markedly elevated creatinine kinase [6••, 32]. Cardiac involvement has been reported in adults with anti-SRP and has also been suggested in affected children [7, 33]. Disease outcomes are poor with one study demonstrating that only half of adult patients reached full or near full-strength after 4 years of treatment [34]. One case series of three JDM patients with antiSRP demonstrated a good response to aggressive treatment with a combination of rituximab, cyclophosphamide and IVIG, which was followed by maintenance methotrexate and intensive daily physical therapy [32].

Two studies have described anti-HMGCR-associated myositis in children. Both reported this to be rare occurring in $\sim$ $1 \%$ of all patients $[35 \bullet, 36 \cdot$. Affected patients were similar to those with anti-SRP; they had more severe muscle disease, very high creatinine kinase levels and were treatment resistant $[35 \bullet, 36 \bullet$. Anti-HMGCR is associated with statin use in adult patients, but affected children had not been exposed to statins
[32, 33]. Interestingly, while anti-HMGCR-associated myositis is generally associated with a good prognosis, statin-naive adults, who are typically younger, may also be refractory to treatment [37].

Although uncommon, IMNM and the associated autoantibodies, anti-SRP and anti-HMGCR, define an important subgroup of JDM patients who frequently present with severe and treatment-resistant disease. The presentation may be perceived as unusual for JDM, where minimal or absent rash and treatment resistance can lead to diagnostic uncertainty [35•]. The identification of a characteristic autoantibody can be helpful to confirm the diagnosis thus facilitating early aggressive treatment and hopefully preventing long-term disability.

\section{Anti-Synthetase Syndrome}

The anti-synthetase syndrome is characterised by myositis, inflammatory arthritis, interstitial lung disease, Raynaud's phenomenon, fever and "mechanics hands"; characteristic skin fissuring at the fingertips. Affected patients have autoantibodies directed against tRNA synthetases, a family of cytoplasmic enzymes responsible for catalysing the binding of amino acids to their corresponding tRNA [38]. There are 20 different tRNA synthetases corresponding to the 20 different amino acids, and, thus far, autoantibodies targeting eight have been described in patients with myositis.

Anti-synthetase syndrome is the most common myositis clinical phenotype in adult patients but is rare in children and found in less than $5 \%$ of those with JDM [6••, 7]. Affected JDM patients are typically older with a median age at disease onset of approximately 14 years, compared to 6.5 years for JDM overall $[6 \bullet \bullet, 7]$. Anti-Jo-1, targeting histidyl tRNA synthetase, is the most common myositis autoantibody in adults and can be identified in $15-30 \%$ of patients $[33,38]$. The remaining anti-tRNA synthetases, anti-PL 7 (threonyl), anti-PL12 (alanyl), anti-OJ (isoleucyl), anti-KS (asparginyl), anti-EJ (glycyl), anti-Zo (phenylalanyl) and anti-Ha (tyrosyl), are rarer, collectively occurring in 10-20\% of adult cases $[33,38]$. While anti-synthetase syndrome is generally viewed as one syndrome, there are established differences between the clinical associations of the different anti-synthetase autoantibodies [29, 39-44].

The anti-synthetase syndrome is comparatively rare in JDM, and, in the UK cohort, anti-synthetase autoantibodies were identified in just six (1.3\%) patients; all patients with an anti-synthetase autoantibody were classified as having JDM overlap and $60 \%$ were of Black ethnicity [3]. Three patients had anti-Jo-1, two anti-PL12 and one anti-PL7 autoantibodies [3]. Importantly, half of this group developed interstitial lung disease, and, interestingly, interstitial lung disease occurred exclusively in those with non-Jo-1 anti-synthetase autoantibodies [6••]. In the adult literature, anti-PL7 and anti-PL12, 
in addition to Black ethnicity, have also been associated with more severe lung involvement [45]. Sabbagh et al. have recently demonstrated that anti-Ro52 in conjunction with an anti-synthetase autoantibody significantly increases the risk of interstitial lung disease in JDM patients [23]. Similarly to our UK cohort, $40 \%$ of JDM patients with an anti-synthetase autoantibody developed interstitial lung disease, but this increased to $100 \%$ of those with anti-Ro52 and an antisynthetase autoantibody [23].

Perhaps because of its relative rarity, the anti-synthetase syndrome is less well-recognised, and JDM patients are commonly labelled as myositis overlap disease [18]. The antisynthetase syndrome is important to identify due to the high incidence of interstitial lung disease [6••, 7, 23].

\section{Overlap Myositis}

Patients can be described as having JDM overlap if they fulfil criteria for both JDM and another rheumatic disease. JDM overlap with systemic sclerosis, inflammatory arthritis and systemic lupus erythematosus have all been widely reported. A potential difficulty with this approach is that rheumatic diseases often have overlapping clinical features. Similarly, the disease phenotype can evolve overtime and affected children can develop features of a related condition as their disease progresses.

Children with anti-synthetase syndrome and anti-MDA5 autoantibodies can be labelled as "myositis overlap" despite the well-characterised myositis disease phenotypes described previously $[18,19]$. We suspect that this is because these myositis subtypes are less well-recognised in children due to their comparative rarity, their association with interstitial lung disease and patients frequently do not have muscle dominant disease.

Anti-PmScl autoantibodies are seen in both patients with myositis and systemic sclerosis, and patients frequently have overlapping features of both diseases. Unlike in adult patients, those with JDM and this autoantibody commonly have dermatomyositis-related skin rashes [6••]. Anti-U1RNP is another autoantibody typical of overlap disease that is common in JDM and particularly older children. Anti-U1RNP is also found in patients with lupus and mixed connective tissue disease. Both these autoantibody subgroups are relatively common in JDM and can be found in 5 and $4 \%$ of UK children, respectively.

\section{Conclusion}

A number of clinically defined subgroups can be seen in patients with juvenile-onset myositis. These subgroups can inform prognosis, further investigations and treatment approach, but the vast majority of patients fall into the classic dermatomyositis category which remains very heterogeneous. Autoantibodies are identifiable in the majority of JDM patients and enable a further degree of phenotypic refinement beyond clinical subgroups. They are particularly helpful in identifying those patients at high risk of interstitial lung disease and who are more likely to respond poorly to standard treatment. Autoantibodies may also help to provide diagnostic clarity, particularly for disease phenotypes that are rare in children.

Acknowledgements We thank Professor Neil McHugh for critical reading of the manuscript.

\section{Compliance with Ethical Standards}

Conflicts of Interest No potential conflicts of interest relevant to this article were reported.

Human and Animal Rights and Informed Consent This article does not contain any studies with human or animal subjects performed by any of the authors.

Open Access This article is licensed under a Creative Commons Attribution 4.0 International License, which permits use, sharing, adaptation, distribution and reproduction in any medium or format, as long as you give appropriate credit to the original author(s) and the source, provide a link to the Creative Commons licence, and indicate if changes were made. The images or other third party material in this article are included in the article's Creative Commons licence, unless indicated otherwise in a credit line to the material. If material is not included in the article's Creative Commons licence and your intended use is not permitted by statutory regulation or exceeds the permitted use, you will need to obtain permission directly from the copyright holder. To view a copy of this licence, visit http://creativecommons.org/licenses/by/4.0/.

\section{References}

Papers of particular interest, published recently, have been highlighted as:

- Of importance

•- Of major importance

1. Symmons DP, Sills JA, Davis SM. The incidence of juvenile dermatomyositis: results from a nation-wide study. Br J Rheumatol. 1995;34(8):732-6.

2. Mendez EP, Lipton R, Ramsey-Goldman R, Roettcher P, Bowyer S, Dyer A, et al. US incidence of juvenile dermatomyositis, 1995 1998: results from the National Institute of Arthritis and Musculoskeletal and Skin Diseases Registry. Arthritis Rheum. 2003;49(3):300-5.

3. Bohan A, Peter JB. Polymyositis and dermatomyositis (first of two parts). N Engl J Med. 1975a;292(7):344-7.

4. Bohan A, Peter JB. Polymyositis and dermatomyositis (second of two parts). N Engl J Med. 1975b;292(8):403-7. 
5. Lundberg IE, Tjarnlund A, Bottai M, Werth VP, Pilkington C, Visser M, et al. 2017 European League Against Rheumatism/ American College of Rheumatology classification criteria for adult and juvenile idiopathic inflammatory myopathies and their major subgroups. Ann Rheum Dis. 2017;76(12):1955-64.

6.• Tansley SL, Simou S, Shaddick G, Betteridge ZE, Almeida B, Gunawardena $\mathrm{H}$, et al. Autoantibodies in juvenile-onset myositis: their diagnostic value and associated clinical phenotype in a large UK cohort. J Autoimmun. 2017a;84:55-64. Description of the autoantibody associated disease phenotypes of a large UK JDM cohort using prospectively collected data

7. Rider LG, Shah M, Mamyrova G, Huber AM, Rice MM, Targoff IN, et al. The myositis autoantibody phenotypes of the juvenile idiopathic inflammatory myopathies. Medicine. 2013;92(4):22343.

8. Iwata N, Nakaseko H, Kohagura T, Yasuoka R, Abe N, Kawabe S, et al. Clinical subsets of juvenile dermatomyositis classified by myositis-specific autoantibodies: experience at a single center in Japan. Mod Rheumatol. 2018:1-6.

9. Deakin CT, Yasin SA, Simou S, Arnold KA, Tansley SL, Betteridge $\mathrm{ZE}$, et al. Muscle biopsy findings in combination with myositisspecific autoantibodies aid prediction of outcomes in juvenile dermatomyositis. Arthritis Rheum. 2016;68(11):2806-16.

10. Yasin SA, Schutz PW, Deakin CT, Sag E, Varsani H, Simou S, et al. Histological heterogeneity in a large clinical cohort of juvenile idiopathic inflammatory myopathy: analysis by myositis autoantibody and pathological features. Neuropathol Appl Neurobiol. 2019;45(5):495-512 A description of the histopathological phenotypes found in a large cohort of JDM patients, including how these relate to autoantibody subgroup.

11. Tansley SL, Betteridge ZE, Shaddick G, Gunawardena H, Arnold $\mathrm{K}$, Wedderburn LR, et al. Calcinosis in juvenile dermatomyositis is influenced by both anti-NXP2 autoantibody status and age at disease onset. Rheumatology. 2014a;53(12):2204-8.

12. Espada G, Maldonado Cocco JA, Fertig N, Oddis CV. Clinical and serologic characterization of an Argentine pediatric myositis cohort: identification of a novel autoantibody (anti-MJ) to a 142-kDa protein. J Rheumatol. 2009;36(11):2547-51.

13. Aouizerate J, De Antonio M, Bader-Meunier B, Barnerias C, Bodemer C, Isapof A, et al. Muscle ischaemia associated with NXP2 autoantibodies: a severe subtype of juvenile dermatomyositis. Rheumatology. 2018;57(5):873-9.

14. Gunawardena H, Wedderburn LR, Chinoy H, Betteridge ZE, North $\mathrm{J}$, Ollier WE, et al. Autoantibodies to a 140-kd protein in juvenile dermatomyositis are associated with calcinosis. Arthritis Rheum. 2009;60(6):1807-14.

15. Orandi AB, Dharnidharka VR, Al-Hammadi N, Baszis KW. Clinical phenotypes and biologic treatment use in juvenile dermatomyositis-associated calcinosis. Pediatric rheumatology online journal. 2018;16(1):84.

16. Bingham A, Mamyrova G, Rother KI, Oral E, Cochran E, Premkumar A, et al. Predictors of acquired lipodystrophy in juvenile-onset dermatomyositis and a gradient of severity. Medicine. 2008;87(2):70-86.

17. Gunawardena H, Wedderburn LR, North J, Betteridge Z, Dunphy J, Chinoy $\mathrm{H}$, et al. Clinical associations of autoantibodies to a p155/140 kDa doublet protein in juvenile dermatomyositis. Rheumatology. 2008;47(3):324-8.

18. Almeida B, Tansley S, Simou S, Gunawardena H, McHugh N, Wedderburn L. Anti-synthetase autoantibody is seen in patients with overlap myositis in the UK cohort of patients with Jveunile Dermatomyositis. Rheumatology. 2017;56(suppl 7):kex390.017.

19. Tansley SL, Betteridge ZE, Gunawardena H, Jacques TS, Owens $\mathrm{CM}$, Pilkington C, et al. Anti-MDA5 autoantibodies in juvenile dermatomyositis identify a distinct clinical phenotype: a prospective cohort study. Arthritis Research \& Therapy. 2014b;16(4):R138.
20. Sato S, Hirakata M, Kuwana M, Suwa A, Inada S, Mimori T, et al. Autoantibodies to a 140-kd polypeptide, CADM-140, in Japanese patients with clinically amyopathic dermatomyositis. Arthritis Rheum. 2005;52(5):1571-6.

21. Kobayashi I, Okura Y, Yamada M, Kawamura N, Kuwana M, Ariga T. Anti-melanoma differentiation-associated gene 5 antibody is a diagnostic and predictive marker for interstitial lung diseases associated with juvenile dermatomyositis. J Pediatr. 2011;158(4):675-7.

22. Kobayashi N, Takezaki S, Kobayashi I, Iwata N, Mori M, Nagai K, et al. Clinical and laboratory features of fatal rapidly progressive interstitial lung disease associated with juvenile dermatomyositis. Rheumatology. 2015;54(5):784-91.

23.• Sabbagh S, Pinal-Fernandez I, Kishi T, Targoff IN, Miller FW, Rider LG, et al. Anti-Ro52 autoantibodies are associated with interstitial lung disease and more severe disease in patients with juvenile myositis. Annals of the rheumatic diseases. Epub ahead of print 2019. Analysis of the impact of anti-Ro52 on phenotype of US JDM patients. An association with ILD and poorer prognosis is described

24. Gerami P, Schope JM, McDonald L, Walling HW, Sontheimer RD. A systematic review of adult-onset clinically amyopathic dermatomyositis (dermatomyositis sine myositis): a missing link within the spectrum of the idiopathic inflammatory myopathies. J Am Acad Dermatol. 2006;54(4):597-613.

25. Bendewald MJ, Wetter DA, Li X, Davis MD. Incidence of dermatomyositis and clinically amyopathic dermatomyositis: a population-based study in Olmsted County. Minnesota Archives of dermatology. 2010;146(1):26-30.

$26 . \bullet$ Betteridge ZE, Gunawardena H, Chinoy H, North J, Ollier WE, Cooper RG, et al. Clinical and human leucocyte antigen class II haplotype associations of autoantibodies to small ubiquitin-like modifier enzyme, a dermatomyositis-specific autoantigen target, in UK Caucasian adult-onset myositis. Ann Rheum Dis. 2009;68(10):1621-5.

27. Bellutti Enders F, Bader-Meunier B, Baildam E, Constantin T, Dolezalova P, Feldman BM, et al. Consensus-based recommendations for the management of juvenile dermatomyositis. Ann Rheum Dis. 2017;76(2):329-40.

28. Hoshino K, Muro Y, Sugiura K, Tomita Y, Nakashima R, Mimori T. Anti-MDA5 and anti-TIF1-gamma antibodies have clinical significance for patients with dermatomyositis. Rheumatology. 2010;49(9): 1726-33.

29. Hamaguchi Y, Fujimoto M, Matsushita T, Kaji K, Komura K, Hasegawa $\mathrm{M}$, et al. Common and distinct clinical features in adult patients with anti-aminoacyl-tRNA synthetase antibodies: heterogeneity within the syndrome. PLoS One. 2013;8(4):e60442.

30. Dalakas MC. Inflammatory muscle diseases. N Engl J Med. 2015;373(4):393-4.

31. Shah M, Mamyrova G, Targoff IN, Huber AM, Malley JD, Rice $\mathrm{MM}$, et al. The clinical phenotypes of the juvenile idiopathic inflammatory myopathies. Medicine. 2013;92(1):25-41.

32. Binns EL, Moraitis E, Maillard S, Tansley S, McHugh N, Jacques TS, et al. Effective induction therapy for anti-SRP associated myositis in childhood: a small case series and review of the literature. Pediatric rheumatology Online Journal. 2017;15(1):77.

33. Betteridge Z, Tansley S, Shaddick G, Chinoy H, Cooper RG, New $\mathrm{RP}$, et al. Frequency, mutual exclusivity and clinical associations of myositis autoantibodies in a combined European cohort of idiopathic inflammatory myopathy patients. J Autoimmun. 2019;101:4855 .

34. Pinal-Fernandez I, Parks C, Werner JL, Albayda J, Paik J, Danoff SK, et al. Longitudinal course of disease in a large cohort of myositis patients with autoantibodies recognizing the signal recognition particle. Arthritis Care Res. 2017a;69(2):263-70.

35. Tansley SL, Betteridge ZE, Simou S, Jacques TS, Pilkington C, Wood M, et al. Anti-HMGCR autoantibodies in juvenile idiopathic 
inflammatory myopathies identify a rare but clinically important subset of patients. J Rheumatol. 2017b;44(4):488-92 A description of 4 JDM patients with anti-HMGCR and a description of the associated phenotype. Although rare, this autoantibody is associated with severe and treatment resistant disease.

36. Kishi T, Rider LG, Pak K, Barillas-Arias L, Henrickson M, McCarthy PL, et al. Association of anti-3-hydroxy-3-methylglutaryl-coenzyme A reductase autoantibodies with DRB1*07:01 and severe myositis in juvenile myositis patients. Arthritis Care Res. 2017;69(7):1088-94. A large study of JDM patients which demonstrates anti-HMGCR to be associated with more severe disease. HLA association is also reported.

37. Mammen AL, Chung T, Christopher-Stine L, Rosen P, Rosen A, Doering KR, et al. Autoantibodies against 3-hydroxy-3methylglutaryl-coenzyme A reductase in patients with statinassociated autoimmune myopathy. Arthritis Rheum. 2011;63(3): 713-21.

38. Mahler M, Miller FW, Fritzler MJ. Idiopathic inflammatory myopathies and the anti-synthetase syndrome: a comprehensive review. Autoimmun Rev. 2014;13(4-5):367-71.

39. Aggarwal R, Cassidy E, Fertig N, Koontz DC, Lucas M, Ascherman DP, et al. Patients with non-Jo-1 anti-tRNA-synthetase autoantibodies have worse survival than Jo-1 positive patients. Ann Rheum Dis. 2014;73(1):227-32.

40. Hervier B, Wallaert B, Hachulla E, Adoue D, Lauque D, Audrain $\mathrm{M}$, et al. Clinical manifestations of anti-synthetase syndrome positive for anti-alanyl-tRNA synthetase (anti-PL12) antibodies: a retrospective study of 17 cases. Rheumatology. 2010;49(5):972-6.

41. Yamasaki Y, Yamada H, Nozaki T, Akaogi J, Nichols C, Lyons R, et al. Unusually high frequency of autoantibodies to PL-7 associated with milder muscle disease in Japanese patients with polymyositis/dermatomyositis. Arthritis Rheum. 2006;54(6):2004-9.

42. Giannini M, Notarnicola A, Dastmalchi M, Lundberg IE, Lopalco G, Iannone F. Heterogeneous clinical spectrum of interstitial lung disease in patients with anti-EJ anti-synthetase syndrome: a case series. Clin Rheumatol. 2016;35(9):2363-7.

43. Hirakata M, Suwa A, Takada T, Sato S, Nagai S, Genth E, et al. Clinical and immunogenetic features of patients with autoantibodies to asparaginyl-transfer RNA synthetase. Arthritis Rheum. 2007;56(4):1295-303.

44. Sato S, Kuwana M, Hirakata M. Clinical characteristics of Japanese patients with anti-OJ (anti-isoleucyl-tRNA synthetase) autoantibodies. Rheumatology. 2007;46(5):842-5.

45. Pinal-Fernandez I, Casal-Dominguez M, Huapaya JA, Albayda J, Paik JJ, Johnson C, et al. A longitudinal cohort study of the antisynthetase syndrome: increased severity of interstitial lung disease in black patients and patients with anti-PL7 and anti-PL12 autoantibodies. Rheumatology. 2017b;56(6):999-1007.

Publisher's Note Springer Nature remains neutral with regard to jurisdictional claims in published maps and institutional affiliations. 